\title{
Wake-induced 'slaloming' response explains exquisite sensitivity of seal whisker-like sensors
}

\author{
Heather R. Beem ${ }^{1,2} \dagger$, Michael S. Triantafyllou ${ }^{1}$ \\ ${ }^{1}$ Department of Mechanical Engineering, Massachusetts Institute of Technology, Cambridge, \\ MA, 02139, USA \\ ${ }^{2}$ Applied Ocean Physics \& Engineering, Woods Hole Oceanographic Institution, Woods Hole, \\ MA, 02543, USA
}

(Received ?; revised ?; accepted ?. - To be entered by editorial office)

Blindfolded harbor seals are able to use their uniquely shaped whiskers to track vortex wakes left by moving animals and identify objects that passed by 30 seconds earlier, an impressive feat as the flow features have velocities as low as $1 \mathrm{~mm} / \mathrm{s}$. The seals sense while swimming, hence their whiskers are sensitive enough to detect small-scale changes in the flow, while rejecting self-generated flow noise. Here we identify and illustrate a novel flow mechanism, causing a large amplitude "slaloming" whisker response, which allows artificial whiskers with the identical unique undulatory geometry as those of the harbor seal to detect the features of minute flow fluctuations when placed within wakes: Whereas in open water the whisker responds with very low amplitude vibration, once within a wake, it oscillates with large amplitude and, importantly, its response frequency coincides with the Strouhal frequency of the upstream cylinder, making the detection of an upstream wake and an estimation of the size and shape of the wake-generating body possible. This mechanism has some similarities with the flow mechanisms observed in actively controlled propulsive foils within upstream wakes and trout swimming behind bluff cylinders in a stream, but there are also differences caused by the unique whisker morphology, which enables it to respond passively and within a much wider parametric range.

\section{Key words:}

\section{Introduction}

Behavioral experiments with harbor seals have demonstrated their outstanding ability to detect and track hydrodynamic signatures left by swimming animals and moving objects (Dehnhardt et al. 2001; Schulte-Pelkum et al. 2007; Wieskotten et al. 2010a,b): figure 1. Despite having their auditory and visual sensory cues blocked, the seals successfully followed the paths of bodies, which had swum ahead of them by 30 seconds or longer. Detection thresholds were measured on the vibrissae (whiskers) of a stationary seal responding to a dipole stimuli in water (Dehnhardt et al. 1998) and reported to be sensitive enough to detect the minute movements left in these hydrodynamic trails.

Various animals utilize similar hair-like structures as direct touch sensors (Barth 2004; Solomon \& Hartmann 2006; Grant et al. 2013). For aquatic animals that swim forward at

$\dagger$ Email address for correspondence: beem@mit.edu 


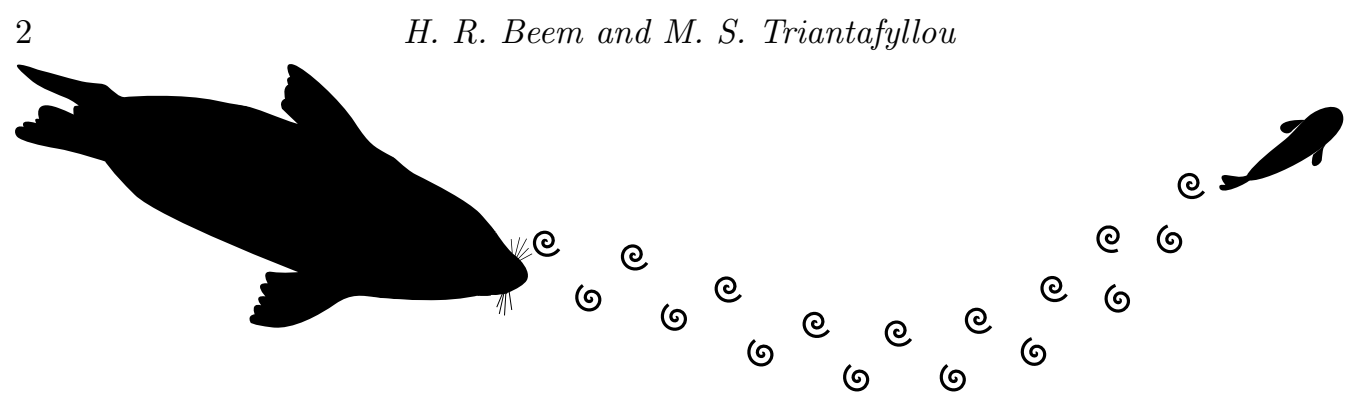

Figure 1: A schematic of a seal chasing a fish by using its whiskers to "feel" for the flow features left in the fish wake.

relatively high speeds, however, it is rather surprising that they would be able to use their whiskers to sense hydrodynamic information. The cross-sectional shape of the whiskers is close in form to a bluff (non-streamlined) body, whose wake is known to spontaneously form a double array of staggered vortices, the Kármán street. The unsteady forces caused by the Kármán street result in vortex-induced vibrations (VIV) on the body, with an amplitude comparable to its cross sectional dimension and with high frequency, resulting in high transverse velocity (Williamson \& Govardhan 2004). Such vibrations would act as strong noise for the sensor, since it would be difficult to discriminate between the wake-induced fluctuations and external flow unsteadiness.

The phenomenon of VIV is ubiquitous for bluff bodies and very complex, as the body vibrations caused by the Kármán street alter, in turn, the vortical structures in the wake and hence the resulting forces and motions, establishing a feedback mechanism that leads to a stable or unstable dynamic equilibrium, which varies significantly as function of the basic parameters of the problem. The frequency of vortex formation $f_{v}$ obeys the Strouhal law, where $D$ is the lateral dimension of the body and $U$ the external flow velocity:

$$
S t=f_{v} D / U
$$

$S t \approx 0.20$ for Reynolds numbers $R e=U D / \nu<250,000$, where $\nu$ is the kinematic viscosity of the fluid (Williamson 1996). In VIV the frequency of vortex formation is entrained by the vibration of the cylinder within a range of non-dimensional frequencies and amplitudes to coincide with the frequency of vibration of the body, hence departing from the Strouhal law. The vortical patterns may resemble a Kármán street, consisting of 2 vortices per cycle ( $2 \mathrm{~S}$ mode), or they may develop two pairs of vortices per cycle (2P mode); other patterns are also possible (Williamson \& Govardhan 2004). As the cylinder oscillates in the cross-flow direction, the added mass term is influenced significantly by the timing of formation of the vortices, whose frequency is synchronized with that of the body; the effective added mass coefficient can vary from a nominal value of 1.0 to a high value exceeding 2.0, or to a low value that can be less than zero. Hence the natural frequency of a flexibly mounted body changes as the effective added mass varies with the parameters of the flow (Williamson \& Govardhan 2004), particularly for mass ratios (ratio of the mass of the body over the displaced fluid mass) close to 1 , as is the case for the vibrissae. Additional complexity is introduced when the body oscillates also in the direction of the current (in-line motion) because the vortical patterns and fluid forces are affected as well (Dahl et al. 2010).

Harbor seals and most other phocid seals have a unique whisker morphology, as described in Hanke et al. (2010) and Ginter et al. (2012). It includes a variable cross-section in the shape of a 2:1 ellipse, whose axes vary sinusoidally in the spanwise direction, while 


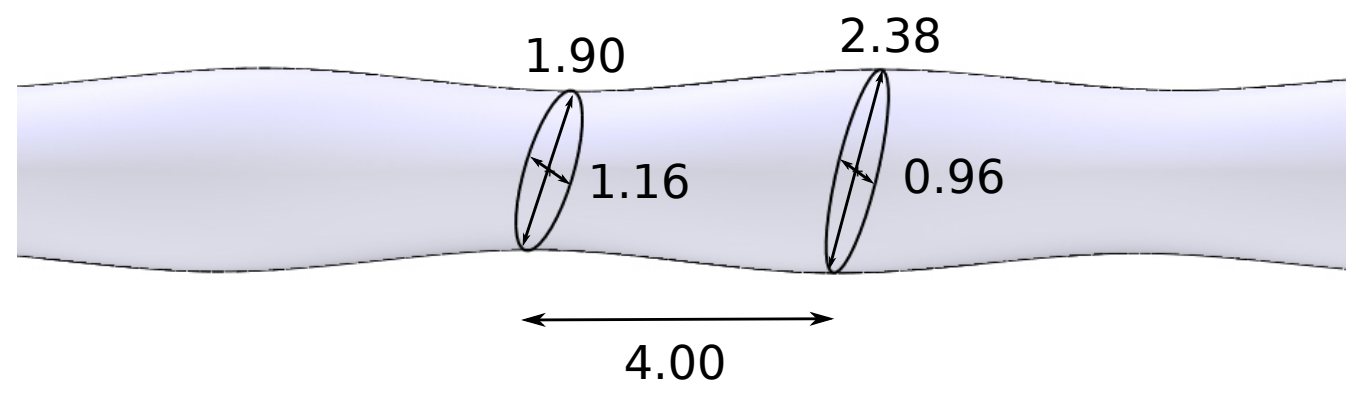

Figure 2: Dimensions of the harbor seal whisker geometry tested in this study. These values (in centimeters) were taken from those provided in Hanke et al. (2010) (termed here "whisker \#1"), and scaled up 20 times. The wavelength of this model ("whisker $\# 2$ ") was modified to have $\lambda / D_{m} \approx 5$, whereas "whisker $\# 1$ " had $\lambda / D_{m} \approx 2$.

the upstream spanwise undulation is not in phase with the downstream undulation. As found in studies of cylinders with periodic variation along the span, such as Ahmed \& Bays-Muchmore (1992); Kim \& Choi (2005); Lam et al. (2004); Lam \& Lin (2009); Lee \& Nguyen (2007); Owen et al. (2000), and Zhang et al. (2005), the whisker also experiences reduced fluid lift and drag when the major axis of the whisker geometry is aligned with the flow (Hanke et al. 2010).

Two versions of this vibrissal geometry are tested in the present study. The first is taken directly from Hanke et al. (2010) and the second, depicted in figure 2, more closely matches both that described in Ginter et al. (2012) and that which the authors measured on specimens provided by the New England Aquarium. The only difference between the two geometries is the wavelength-to-diameter ratio: $\lambda / D_{m} \approx 2$ in the first and $\lambda / D_{m} \approx 5$ in the second, where $D_{m}$ is the mean overall diameter.

Flow simulations reveal that these undulations break the spatial coherence of Kármán vortex formation and cause the formation of streamwise vorticity, hence reducing substantially the fluid forces on the whisker (Hanke et al. 2010). This feature is believed to allow the seal to move forward at steady speed with small self-induced noise from VIV. The question remains on how the vibrissae detect unsteady velocities, especially the minute velocities found in wakes $30 \mathrm{~s}$ after a body has passed (Wieskotten et al. $2010 a)$.

To answer this question, we turn to a simplified problem that contains the main flow features in fish wake detection: A flexibly mounted whisker is placed in the wake of an upstream cylinder that generates coherent vortices, thus subjecting the whisker to oscillatory forces. Seals swimming in the wakes of fish encounter a more complex flow, which is either momentum-less, when the fish are in steady motion, or with positive thrust, when the fish are accelerating. In both cases, however, the wake contains a strong jet, as seen in visualizations behind fish (Müller et al. 1997; Wolfgang et al. 1999). The body and caudal fin generate coherent ring-like vortical structures that induce a jet flow, as well as a drag wake, and it is only in the very far field when the two cancel each other for a self-propelled body. Visualization on planar cuts through these wakes show the predominance of a flow that closely resembles a reverse Kármán street - which has the same features as a regular Kármán street, but the vortices rotate in opposite direction. Hence the present model holds relevance to the fish detection problem, has a much simpler form, and can draw on the extensive knowledge of the hydrodynamic properties of interfering cylinders. Still, seals have been shown to also detect differently shaped wakes, such as those of propeller-driven miniature submarines (Wieskotten et al. 
$2010 b$ ), where helical vortices form by the propeller; so this study is only a first step towards a full mapping of the detection properties of seal whiskers.

We look, then, to the problem of wake-induced oscillations, which occur when a flexibly mounted body is placed in the wake of an upstream body, so it is subject to the unsteady forcing of its vortical structures. Most research has addressed the problem of two interacting circular cylinders of equal or comparable diameter (Hover \& Triantafyllou 2001; Papaioannou et al. 2006; Assi et al. 2006, 2010), in which it was shown that the upstream cylinder has significant effect on the amplitude and frequency of motion of the downstream cylinder even when placed 25 diameters apart. The upstream cylinder continues to have significant effect on the downstream cylinder's response when the two are of unequal diameter and at relatively small distances apart (Sayers \& Saban 1994; Lam \& To 2003; Assi 2014b; Gao et al. 2014). It was also found that a 'wake stiffness' exists - that is, the vortical wake of the upstream cylinder causes a restoring force on the downstream cylinder, which is equivalent to a linear spring, hence affecting its natural frequency (Assi et al. 2013). No studies for large distances are available.

In this paper we employ a flexibly mounted model of a seal whisker placed within the wake of a larger upstream circular cylinder, modeling the response of a single seal whisker within a wake produced by another object. Strain measurements and dye visualizations are used to elucidate the whisker's mechanism of detecting the features of the unsteady flow. Our principal aim is to explain the ability of seals to detect far wakes fluctuating with minute velocities, of the order of $1 \mathrm{~mm} / \mathrm{s}$, and hence track the wake-producing bodies, while moving at speeds of the order of $1 \mathrm{~m} / \mathrm{s}$. We call this exquisite sensitivity, since the ratio of detectable velocity to the whisker's own forward velocity can be of the order of 1,000, and wake feature recognition requires identification of the dominant frequencies of the minute unsteady fluctuations of the wake. We identify and describe a mechanism of flow energy extraction that drives strong oscillations of the whiskers, leading to a 'slaloming motion' whose frequency is locked to the dominant frequency of the oncoming wake, even at large distances from the upstream object.

\section{Methodology}

\subsection{Experimental setup}

The whisker model was mounted in its streamlined direction (angle of attack $\alpha=0^{\circ}$ ) and in a vertical position in the MIT Towing Tank, fixed at its top on a spring-like structure, which allowed it to freely move in cross-flow and in-line vibrations, as explained in detail in the next section. The VIV response was first tested by towing it in calm water. Next, a hydrodynamic wake was generated upstream of the whisker model through the use of a vertically mounted cylinder, which shed a Kármán vortex street (figure 3). For comparison purposes, these tests were repeated with a smooth elliptical (2:1 diameter ratio) cylinder model and then a circular cylinder model, both with the same mean cross-flow diameter $\left(d_{w}\right)$ and length $L$ as the whisker \#1 model.

In the case of the harbor seal, the whisker diameter, which is around 0.5 to $1 \mathrm{~mm}$, is typically much smaller than the dimension of the dominant wake vortices, which scale with the dimension of the upstream body. The diameter of the upstream cylinder was therefore chosen to be larger than that of the whisker model, taking values $d_{c y l}=\{2.5,4,11\} d_{w}$. The two objects were towed simultaneously along the tank at velocity $U$. The whisker's amplitude and frequency of vibration were measured throughout. To assess the effect of the distance between the two objects, the tests were conducted at four different down- 


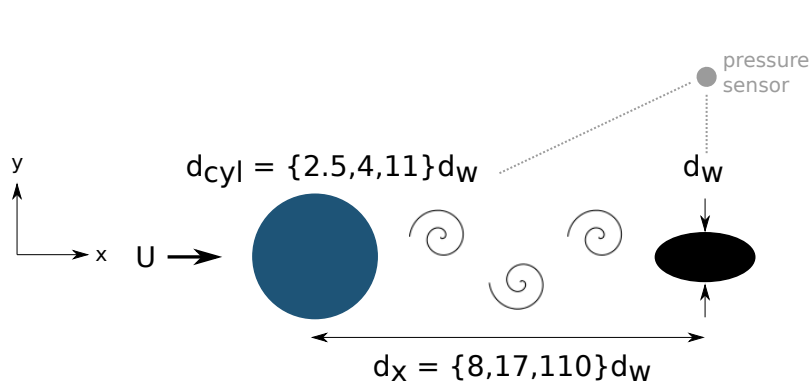

top view

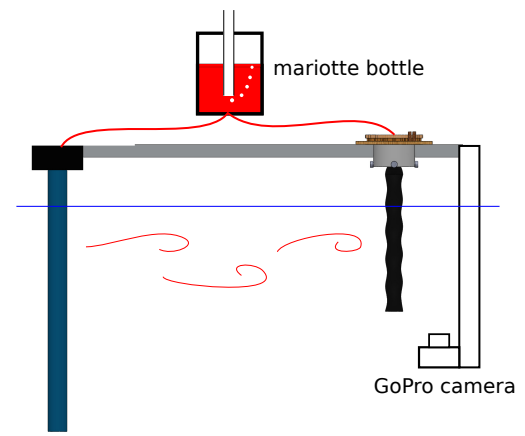

side view

Figure 3: Left: top; and right: side views of the setup, showing the generation of a hydrodynamic trail in front of the whisker through the use of a cylinder that generates a Kármán vortex street. Four different downstream distances $\left(d_{x}\right)$ were tested, ranging from the near wake to the far wake. Three different cylinder sizes $\left(d_{c y l}\right)$ were also tested. Simultaneous measurement of the oncoming wake frequency and whisker response was done through use of a pressure sensor placed near the edge of the wake. A mariotte bottle supplied dye at a constant flow rate. A camera underneath the model captured a bottom-up view of the wake-whisker interaction.

stream distances $\left(d_{x}=\{8,17,110,160\} d_{w}\right)$, representing cases of close proximity, moderately large distance, and large distances, respectively.

Also, the principal flow features of the upstream wake were measured directly and in real-time during the tests. This was done by placing a pressure sensor (Freescale MPXV7007, on-chip conditioning) at the same downstream location as the whisker to collect information on the cylinder wake. In order to avoid interfering with the flow encountered by the whisker, the pressure sensor was placed at a lateral location closer to the edge of the wake (at an angle of $60-75^{\circ}$ between the upstream cylinder and the whisker). Plastic tubing was connected to the sensor inlet and placed into the wake, such that the sensor avoided direct contact with water. To reduce flow-induced vibrations of the tube itself, it was attached to the leading edge of a small airfoil wing, which was rigidly mounted to the carriage. A National Instruments USB-6211 DAQ board was used to collect the analog signal (sampling frequency $=1000 \mathrm{~Hz}$ ). The dominant frequency of the wake was extracted from the pressure signal and compared with that of the whisker oscillation.

\subsection{Whisker-like sensor design}

A model whisker was fabricated out of plastic using stereolithography. A free vibration setup (figure 4) was designed by mounting the base of the model on a flexing plate (1095 spring steel, thickness $=0.38 \mathrm{~mm}$ ) with four arms, allowing the model to freely vibrate in the in-line $(x)$ and cross-flow $(y)$ directions. It was therefore in a cantilevered configuration, resembling that on the seal, although the main body of the whisker remains rigid in the experiment.

Note that bushing/pin assemblies serve to both hold the end of the flexures in place and reduce coupling between the $x$ and $y$ axes. As the plates in one axis deflect, the bushings on the off-axis rotate, preventing the off-axis plates from deflecting unnecessarily. If strong coupling is allowed, large vibrations are noted.

Strain gauges (Omega, Model KFH-03-120) on the flexing plates were arranged in a 
Wheatstone bridge to output a signal proportional to the deflection of the plates. Strain signals were converted to a voltage once acquired through a PhidgetBridge 1046 USB Input Board (sampling frequency $=60 \mathrm{~Hz}$, gain $=16$ ). The measurements collected were calibrated to the amount of deflection at the tip of the model. Calibration curves relating voltage and tip deflection were found to be linear for both axes on all models, with the lowest coefficient of determination $\left(R^{2}\right)$ value being 0.953 .

The natural frequency $\left(f_{n}\right)$ of each model was found by conducting pluck tests in water and calculating the zero-crossing frequency of the signals. The whisker \#1 and elliptical cylinder models have a natural frequency of $f_{n, x}=3.1 \mathrm{~Hz}$ in-line and $f_{n, y}=2.2 \mathrm{~Hz}$ in cross-flow. The whisker \#2 model was designed to have similar mass to these two, allowing it to have similar natural frequencies $\left(f_{n, x}=3 \mathrm{~Hz}, f_{n, y}=1.8 \mathrm{~Hz}\right)$. The circular cylinder model has a smaller mass relative to the other models, giving it a lower frequency $\left(f_{n}=1.8 \mathrm{~Hz}\right)$, which also necessitated the use of a slightly thinner flexure (1095 spring steel, thickness $=0.2 \mathrm{~mm}$ ) to cover an appropriate non-dimensional towing velocity $\left(U^{*}=U /\left(f_{n, y} d_{w}\right)\right)$ range. Pluck tests in air reveal the models' structural damping to range from $\zeta=0.5 \%$ to $2 \%$, represented as a percent of the critical damping. Testing of seal vibrissae (Hans et al. 2014) shows that damping of individual vibrissae is small to negligible, although it is not known whether animals actively control the effective stiffness and damping of the vibrissae by adjusting the stiffness of their cheeks.

All four models have a mass ratio $\left(m^{*}=m / m_{d}\right)$ of 1.4 , where $m$ is the model mass and $m_{d}=\rho \pi \frac{d_{w}^{2}}{4}$ span is the displaced fluid mass. Due to experimental constraints, the whisker \#2 model was fabricated at a 20 times scale version of the real whisker dimensions (resulting in $d_{w}=1.06 \mathrm{~cm}$ ) and has a submerged span of $L / d_{w}=30$, whereas the other models are at a 30 times scale $\left(d_{w}=1.59 \mathrm{~cm}\right)$ and have $L / d_{w}=12$. The Reynolds number based on the diameter $d_{w}$ ranges from 1,060 to 20,670, given the range of $\mathrm{U}$ covering 0.1 to $1.3 \mathrm{~m} / \mathrm{s}$, so it is generally larger than the Reynolds number of seal whiskers which is around 1,000. The higher Reynolds number in the experiments allows easier measurement of forces and motions.

\subsection{Experimental Data Analysis}

The strain signals were recorded as function of time for each run. A bandpass Butterworth filter (pass range: 0.4 to $20 \mathrm{~Hz}$ ) was applied. To exclude transient phenomena, only a middle segment of these signals was considered further. The vibration amplitude for that run was determined by taking the root-mean-square (RMS) amplitude of that segment. The value was then non-dimensionalized by $d_{w}$. The non-dimensional crossflow frequency of vibration was determined by taking a fast Fourier transform (FFT) of the signal, selecting the peak response frequency, and dividing it by the model's natural frequency in water: $f_{y}^{*}=f_{y} / f_{n, y}$.

Finally, the data utilizing simultaneous measurements of wake pressure and strain on the whisker sensor was analyzed over a finer time scale. First, the two signals were resampled to the same frequency and then the following signal conditioning was applied to both signals. A bandpass Butterworth filter was used to pass frequencies between 1/2 and 2 times the expected wake frequency, based on the Strouhal relation. Additional Butterworth filters were used to notch out the natural frequencies of the foil $(\approx 1,10,20$ $\mathrm{Hz}$ ). Cross-correlation of the two signals was conducted to quantify any similarity. This correlation was first conducted by dividing the signals by their RMS value in order to normalize their magnitudes. An initial cross-correlation was carried out to determine the value of any time delay between the two signals. The signals were shifted by a delay corresponding to the peak in this initial cross-correlation result. A second cross-correlation was then used to determine the level of synchronization between the two signals. This 


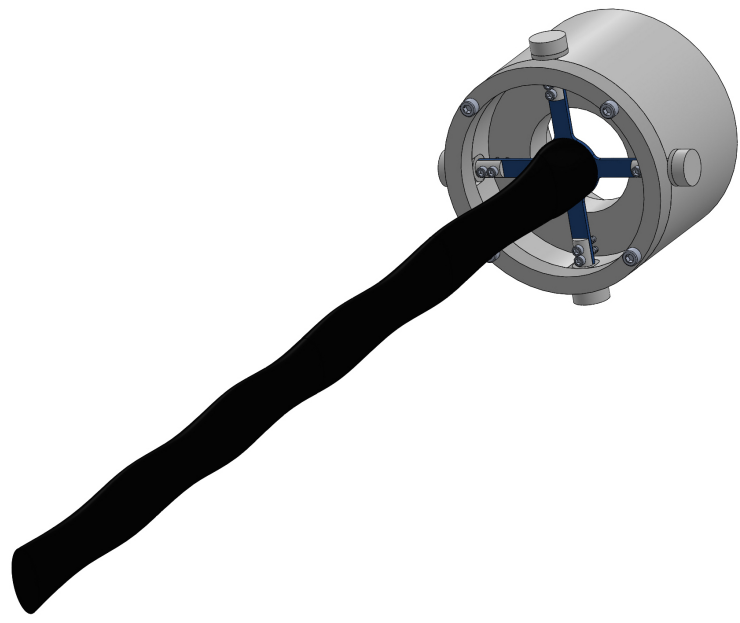

(a)

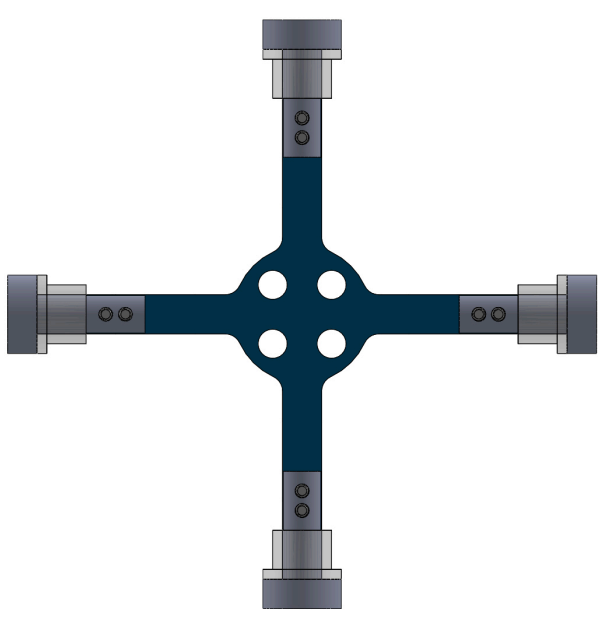

(b)

Figure 4: (a) The model whisker is mounted on a four-armed spring steel flexure that allows free vibration in in-line and cross-flow directions. (b) Bushings and pins at the end of each arm allow for free rotation in the direction that is not being deflected, which reduces cross-coupling.

value was normalized by the magnitude to reach a cross-correlation coefficient $(c)$, which is the variable presented in the following plots.

\subsection{Flow visualization apparatus}

A mariotte bottle setup was constructed in order to introduce dye into the flow for visualizations. This system is made of a sealed container of dye with an air inlet on the top and a dye outlet on the bottom (figure 3). The air inlet ensures that the pressure inside the bottle remains constant even as the reservoir level lowers. The dye can therefore be delivered at a constant rate. The tracer was made by combining 8 parts water, 5 parts industrial food coloring, and 1 part alcohol, which resulted in a neutrally buoyant liquid.

Under open water conditions, a tube from the mariotte bottle extends into the top of the model, allowing the dye to exit through a small hole on the downstream side of the model at the $65 \%$ span level. When the model was placed in the wake of an upstream cylinder, the dye was introduced into the flow between the wake generator and the whisker. The tube from the mariotte bottle was placed into the wake generator (a hollow PVC pipe with a plug at the bottom) and dye exits from a small hole on the downstream side of the cylinder, again at the $65 \%$ span level of the whisker. An underwater camera (GoPro Hero 3 Black Edition) was mounted below the whisker, providing a bottomup view. It was positioned sufficiently below and downstream of the model to avoid disturbing the flow seen by the model. Videos of the cylinder wake-whisker interaction were captured at $1440 p$ resolution and at 48 frames per second. Videos of the wake behind the models moving through calm water were captured at $720 p$ resolution, 120 frames per second. 


\section{Whisker-like sensor responses and flow visualization}

\subsection{Vortex-induced response in open flow}

First, the vortex-induced vibrations of each model were measured using strain gauges as the models were towed through open water (no upstream body). The whiskers and elliptical cylinder were oriented with their long axis aligned with the flow direction. Figure 5(a) shows the non-dimensional tip deflection for all models in the cross-flow direction $\left(A_{y}^{*}=A_{y} / d_{w}\right)$ and in the in-line direction $\left(A_{x}^{*}=A_{x} / d_{w}\right)$ versus the reduced velocity $\left(U^{*}\right)$. The non-dimensional frequency of cross-flow oscillation $\left(f_{y}^{*}=f_{y} / f_{n, y}\right)$ is also plotted.

Flemming \& Williamson (2005) and Assi (2014a) tested circular cylinders in a similar configuration as in our experiments, viz. pivoted at the top, freely vibrating in the inline and cross-flow directions and found a peak amplitude of vibration of $\sim 1.5 d$ at the tip, similar to the value found here. The elliptical cylinder and whisker experience very low VIV compared to a circular cylinder. Also, the oscillation frequency for the elliptical cylinder and whisker remain close to their natural frequency across all speeds tested. In previous seal whisker vibration studies (Hanke et al. 2010; Murphy et al. 2013), the additional degree-of-freedom in the in-line direction was not included. Motion in the inline axis can induce additional vibrations (Dahl et al. 2010). In spite of this added degree of freedom, the whisker VIV was found to remain low in our experiments.

Figure 5(b) shows a zoomed-in view of the cross-flow response. The elliptical cylinder exhibits similar but slightly higher vibrations than the whisker. The whisker \#2 model has an even lower response than the original model. Note that the difference in Reynolds number in minor (data here from whisker \#1 is at $R e=2,120$ to 12,720 and whisker \#2 is at $R e=1,590$ to 11,130$)$. These two geometry variations may align with the two minima seen in Lam \& Lin (2009).

Dye visualizations of the $0^{\circ}$ configuration illuminate wake patterns that explain the recorded deflection measurements. A circular cylinder sheds large coherent vortices in a $2 \mathrm{~S}$ pattern. The elliptical cylinder also sheds coherent vortices but with a narrower wake. Whisker \#1 displays a less coherent vortex wake that forms further downstream. The whisker \#2 model (not shown here) creates a similar wake as whisker \#1.

Due to the improved VIV reduction performance of the second whisker model, the remainder of this paper focuses on its properties. If the model number is not explicitly mentioned, it should be taken to refer to the whisker \#2 model.

\subsection{Whisker responses when placed within an upstream wake}

We now consider the case where the whisker is at a relatively close distance from the upstream cylinder, $d_{x}=8 d_{w}$, when the cylinder diameter is two and a half times larger than the whisker's, $\left(d_{c y l}=2.5 d_{w}\right)$. The whisker is still facing the flow along its streamlined direction $\left(\alpha=0^{\circ}\right)$.

The whisker's non-dimensional amplitude of vibratory response at the tip is shown in figure $7 \mathrm{a}$. The amplitude in this case peaks at a value $>1.5 d_{w}$. This value has to be contrasted with the whisker's vibration amplitude as it moves through open water (depicted with the " $\mathrm{x}$ "'s); the latter is significantly lower - peaking at $\sim 0.05 d_{w}$. Hence, for this close distance and size ratio, the whisker vibrates with 30 times larger amplitude when it encounters the wake than it does in open water. The same whisker placed at a large distance away from the cylinder, $d_{x}=160 d_{w}$, the peak vibration remains high, reaching $>3$ times the value in open water. As above, the bars denote the data spread over three repeat runs.

Also of note is the comparison of the whisker's frequency of response between the 


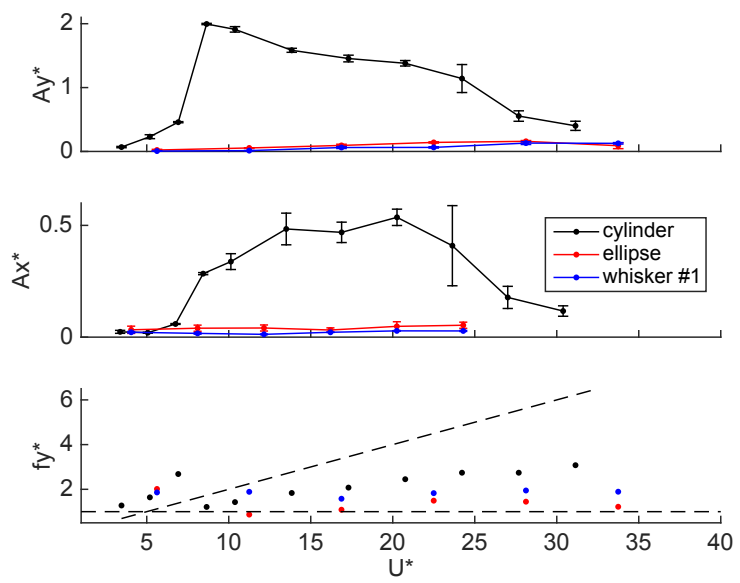

(a)

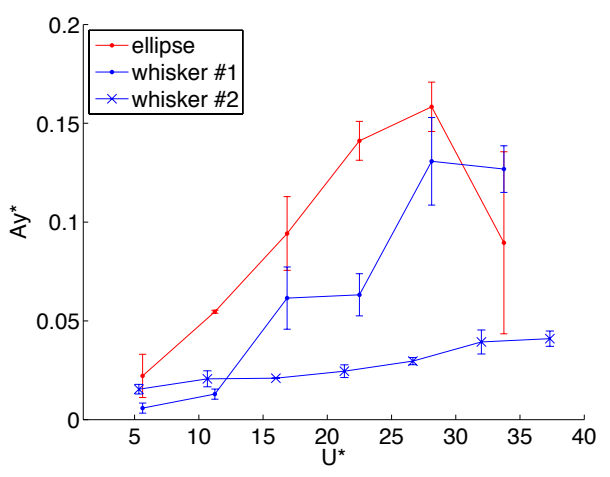

(b)

Figure 5: VIV response in open flow of the whisker, elliptical cylinder (ellipse), and circular cylinder; the first two models positioned along their streamlined direction. Bars depict data spread over three repeat runs. (a) Both cross-flow and in-line amplitudes of the whisker and elliptical cylinder are significantly smaller than those of the circular cylinder. The frequencies at which the whisker and ellipse vibrate remain close to their natural frequency. The diagonal dotted line indicates a frequency that matches $S t=0.2$. (b) Both whiskers and the elliptical cylinder vibrate with $A_{y}^{*}<0.2$. Whisker \#2 has the lowest VIV amplitude.
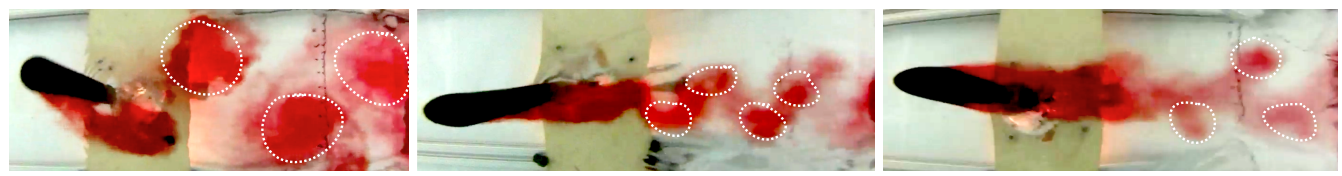

Figure 6: The wake behind a circular cylinder, elliptical cylinder, and whisker (from left to right) positioned along their streamlined direction $\left(\alpha=0^{\circ}\right)$ is visualized using dye. The first two objects shed regular $2 \mathrm{~S}$ patterns, whereas the whisker \#1 model sheds an incoherent wake. Images were taken during runs corresponding to each model's peak amplitude: cylinder at $U^{*}=10$, ellipse at $U^{*}=28$, and whisker $\# 1$ at $U^{*}=28$.

two cases, shown in figure $7 \mathrm{~b}$, where the non-dimensional frequency of vibration $\left(f_{y}^{*}\right)$ is plotted versus $U^{*}$. When the whisker moves through open water, it oscillates at small amplitude and with a relatively constant frequency. However, when the whisker encounters an oncoming vortical wake, it oscillates at a new frequency, $f_{y}$, that matches the vortex shedding frequency of the upstream cylinder, as measured by the pressure sensor during the same runs. Both frequencies are non-dimensionalized by the whisker's natural frequency and plotted together. Hence the whisker is shown to synchronize with the oncoming wake dominant frequency, which is distinct from its VIV frequency in open water. Similar frequency synchronization was reported in Miersch et al. (2011) for a rigidly-mounted whisker. The frequency response of the whisker at large distance is discussed in detail in section 3.4.

The large amplification of the vibratory response and the lock-in of the whisker response to the Kármán frequency constitute a mechanism of wake detection that the seals can use: The whiskers, which normally vibrate at very low amplitude when in open water, 

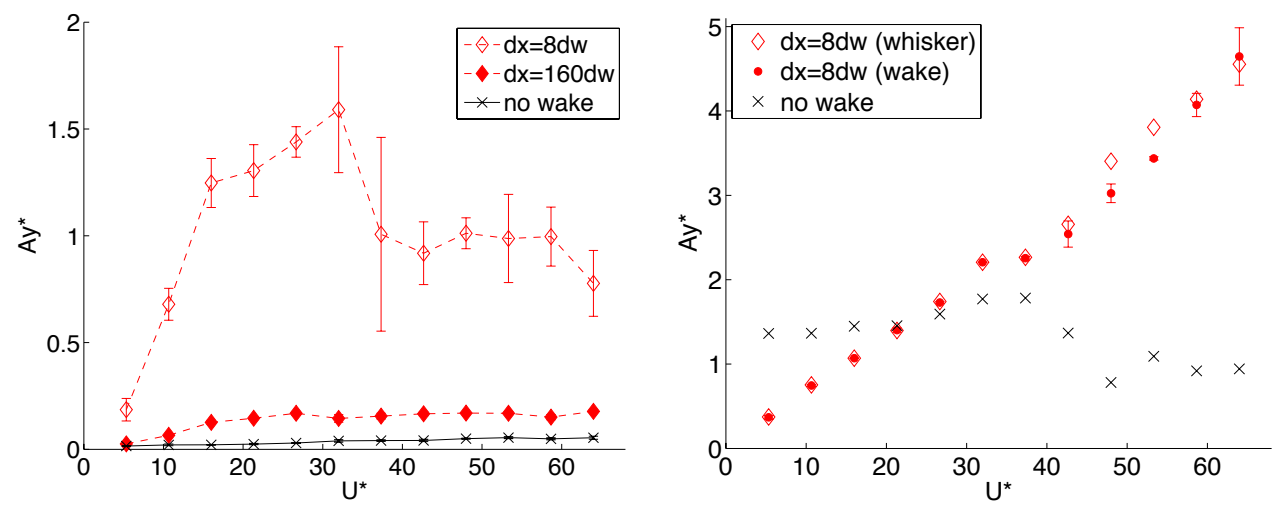

Figure 7: For a whisker placed in the near wake of a larger diameter upstream cylinder $\left(d_{c y l}=2.5 d_{w}\right)$, at a distance $d_{x}=8 d_{w}$ : (Left) The whisker vibrates with a far larger amplitude when placed within the wake than in open flow. (Right) The peak frequency of the whisker's vibration synchronizes with the wake's vortex formation frequency. Each symbol represents the average over three repeat runs and data spread is depicted through bars.

vibrate with an amplitude that are an order-of-magnitude larger when in the wake of a body; and, more importantly, the frequency of vibration synchronizes with the dominant frequency in the wake.

\subsection{Flow visualization of a whisker within an upstream wake}

Flow visualizations of the whisker interacting with the wake provide the flow mechanism that explains these findings. Videos are taken with a bottom-up perspective, under similar conditions: $d_{c y l}=2.5 d_{w}, d_{x}=17 d_{w}$, and displayed in a sequence of snapshots (figure 8). Dye exits the wake generator, off the right side of images, is entrained by the vortices shed by the cylinder and then impinges on the whisker. The whisker is painted white, while the whisker cross-section that lies within the dye plane (at $65 \%$ of the whisker span) is outlined. The principal feature is a "slaloming" trajectory, whereby the whisker synchronizes its motion to come very close to, but never piercing the nearest vortex within the Kármán street, and then, as it moves further downstream, moves rapidly away from that vortex, to encounter and get close to the next vortex, at the opposite side of the Kármán street, and so on. The pressure gradient characterizing each vortex provides the suction force driving the whisker oscillations with amplitude comparable to the width of the wake, and causing synchronization with the dominant wake frequency. This constitutes the basic flow energy extraction mechanism.

\subsection{Effects of distance and geometry}

For comparison purposes, the same tests are repeated using, instead of the whisker, the elliptical and circular cylinders at multiple downstream distances. The amplitude values are compared in figure 9 with the circular cylinder on the left, elliptical cylinder in the middle, and whisker on the right. Results from the three different separation distances are shown, as well as the response in open water.

At the two closest distances, all three shapes show signs of wake induced response similar to trends reported in the literature for cylinders in tandem at similar distances: $d_{x} / d=2$ to 5.6 (Hover \& Triantafyllou 2001; Assi et al. 2006, 2010, 2013). The circular 


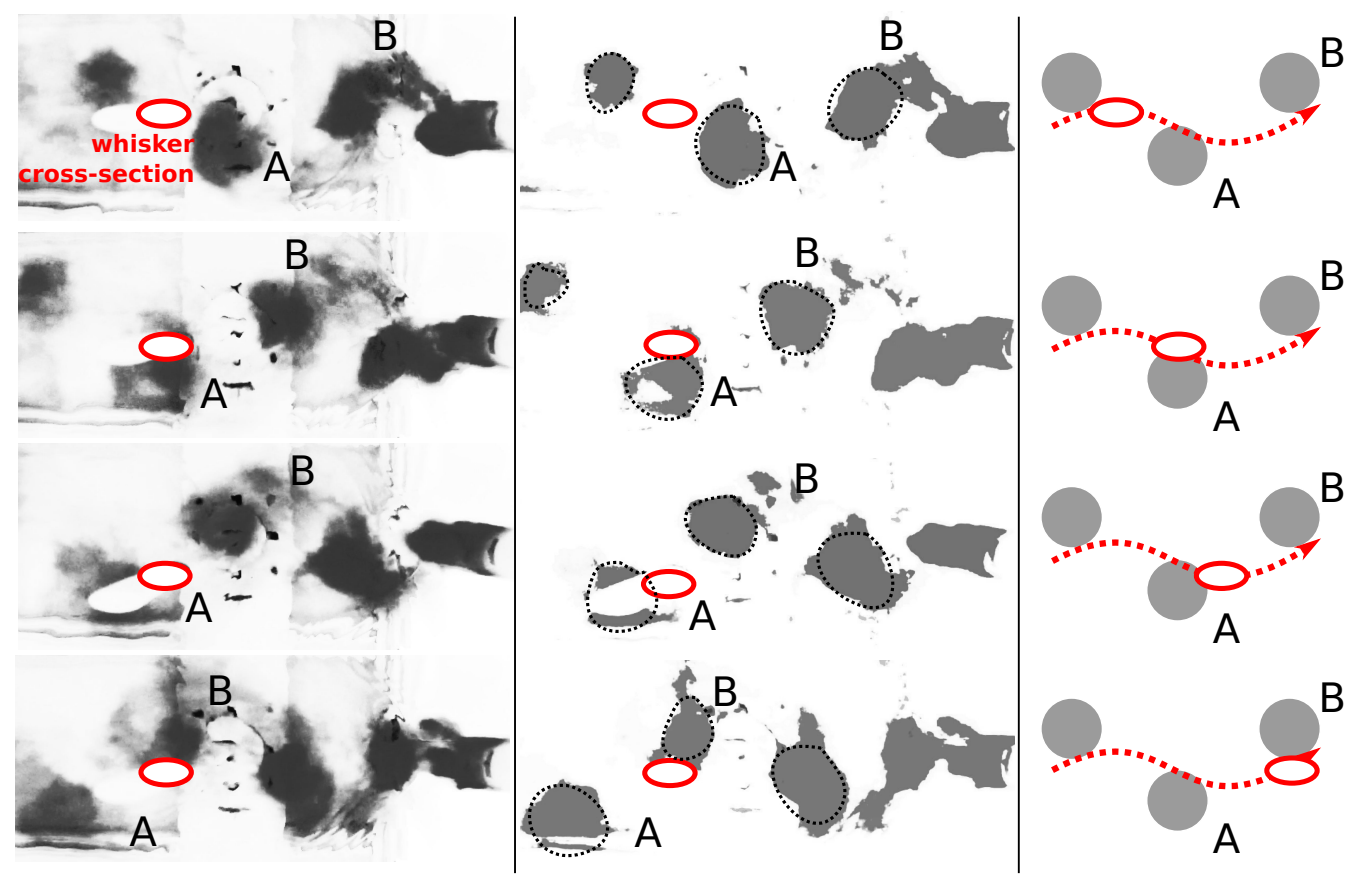

Figure 8: Dye visualization sequence of a whisker placed within a wake (left: original image; center: high contrast image) depicting the location of the oncoming vortices relative to the cross-section of the whisker (outlined in red at the dye plane); and schematic (right) depicting the whisker's "slaloming" free vibration path through the vortex wake. The frequency of the whisker's motion locks in with the vortex wake frequency, as the whisker slaloms between the Kármán vortices. $d_{c y l}=2.5 d_{w}, d_{x}=17 d_{w}, U^{*}=16, R e=3,180$ )

cylinder vibrates at roughly similar amplitudes in the wake as it does in open water (except at the closest distance). The elliptical cylinder and whisker, on the other hand, oscillate substantially more as they encounter the wake. These amplitudes largely decrease as the downstream distance increases. Finally, unlike the other two models, the whisker continues to vibrate with amplitude significantly higher than its VIV response at the farthest distance $\left(160 d_{w}\right)$. The wake-induced vibration (WIV) amplitude of the whisker at that distance reaches $\sim 4$ times higher than the VIV in open water, indicating the whisker's superior ability to detect a wake at a far distance.

The peak frequencies of these responses are compared to the theoretical frequency of the wake, based on a Strouhal number of 0.2 , and shown in figure 10 with $d_{x}$ increasing from left to right. At the closest distance, all models synchronize with the theoretical wake frequency. At the medium distance, the cylinder responds with a frequency that is consistently different than the theoretical, as the wake stiffness would dictate. At a large distance, the whisker and elliptical cylinder align with the theoretical wake frequency, but only over a relatively short range of reduced velocities. It should be noted, also, that the far wake develops new vortical patterns that are different than the originally patterns shed close to the cylinder (Williamson 1996).

\subsection{Effect of cylinder relative size}

The effect of the relative size of the wake generator on the detection ability of the whisker was tested by using three different size cylinders with diameter $\left(d_{c y l}=\{2.5,4,11\} d_{w}\right)$, 


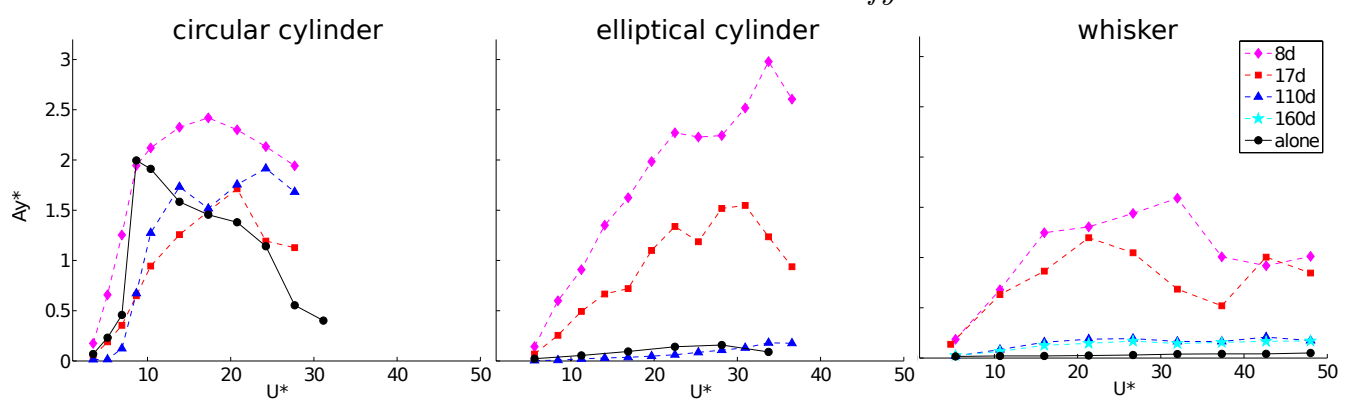

Figure 9: Comparison of the vibration amplitudes of the circular cylinder, elliptical cylinder, and whisker (from left to right, respectively). The latter two vibrate significantly more when they are in the wake of an upstream cylinder (colored lines) than they do in open water. The circular cylinder vibrates with a similar amplitude for all cases. The amplitudes of response decrease as the distance from the upstream cylinder increases. The whisker retains an amplitude greater than its VIV amplitude even at the largest distance tested. Each symbol is the average of three repeat runs.

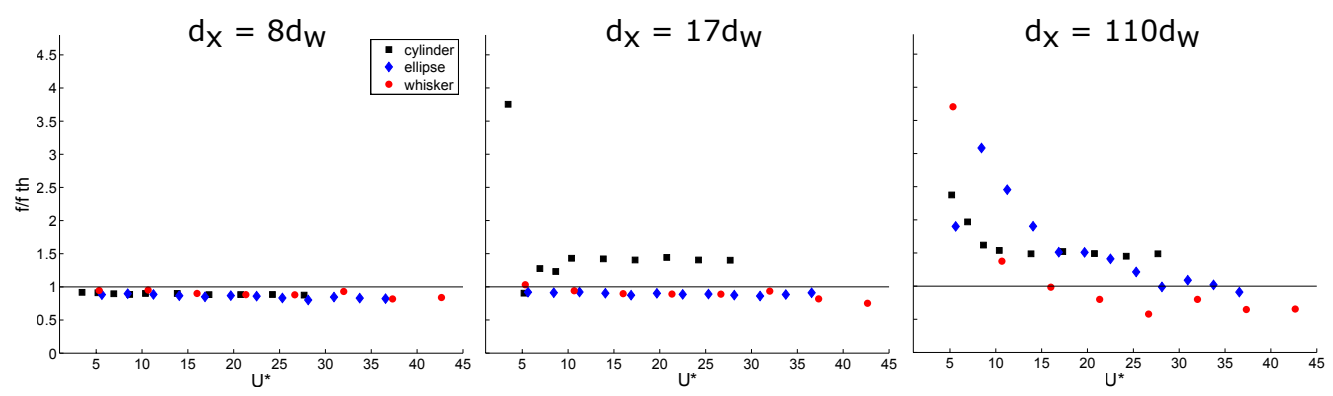

Figure 10: Peak vibration frequencies of response for the circular cylinder (black square), elliptical cylinder (blue diamond), and whisker (red circle) at three different distances behind a cylinder $\left(d_{c y l}=4 d_{w}\right)$. At small distance $\left(8 d_{w}\right)$, all three models vibrate at the theoretical wake frequency. As the distance increases to $17 d_{w}$, the elliptical cylinder and whisker continue to oscillate at the wake frequency, but the cylinder deviates from it. At a large distance $\left(110 d_{w}\right)$, only the elliptical cylinder and whisker have peak frequencies that stay close to the theoretical wake frequency over a range of reduced velocities. Each symbol is the average of three repeat runs.

respectively. In order to ensure similarity, the whisker was placed at the same downstream distance, measured in cylinder diameters $d_{x}=15 d_{c y l}$.

The cross-correlation coefficient $(c)$ between the whisker's response signal and the pressure signal from the upstream wake was calculated. A cross-correlation value of 1 indicates perfect synchronization between the whisker and the wake frequencies, and a value of 0 indicates no synchronization. In figure 11(a), the maximum cross-correlation coefficient per run is plotted. For all sizes of cylinders tested, there is a high $(>0.85)$ maximum value.

Wakes can be intermittent, especially at large distances, and hence synchronization does not last for the entire run. Even though the peak correlation was high in all runs, as shown in figure 10, the whisker frequency matches the expected frequency over the entire run only in some cases. Hence, we will refer to a "sufficient synchronization" when the instantaneous cross-correlation coefficient (measured over one oscillation cycle) has 

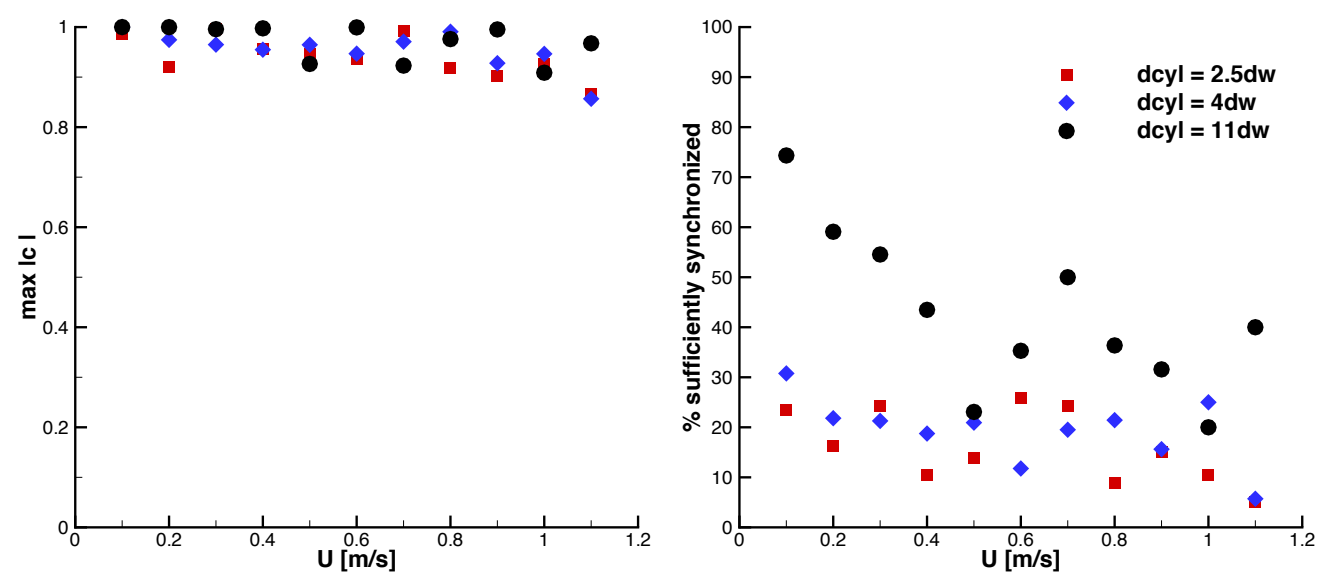

Figure 11: Cross-correlation of the whisker vibration with the wake oscillations for three different size cylinders at $d_{x}=15 d_{c y l}: d_{c y l} / d_{w}=2.5$ (red square), 4 (blue diamond), 11 (black circle). (Left) The cross-correlation coefficient between the whisker vibration and upstream wake signals reaches a high $(>0.85)$ maximum value for all speeds and cylinder sizes tested. (Right) The percentage of the run where the whisker is "sufficiently synchronized" (defined as $c>0.8$ or $c<-0.8$ ) generally increases with cylinder diameter. Hence, the whisker's detection ability increases with the size of the wake features.

absolute value $>0.8$. The duration of time over which the whisker remains "sufficiently synchronized", shown in figure 11b, indicates that even at large distances the whiskers are able to lock-in with the frequency of the wake for some period of time that could enable wake detection. Also, as the diameter of the cylinder increases, and therefore the size of the dominant wake features increase, the percentage of the run where the whisker is "sufficiently synchronized" generally increases.

\section{Discussion}

Observations on flow detectability in experiments using trained seals (Dehnhardt et al. 2001; Schulte-Pelkum et al. 2007; Wieskotten et al. 2010a) have established the impressive performance of whiskers as minute wake flow detectors. This paper builds upon these findings to determine some principal mechanisms that enable the fine sensitivity of the whiskers even as the animals move at relatively high speed. We have used a simplified setting- that of a whisker in the wake of a larger-diameter upstream cylinder. As explained in the introduction, because the wakes of fish contain organized, high aspect ratio, ringlike vortical structures (Müller et al. 1997; Wolfgang et al. 1999), which appear in planar cuts as reverse Kármán streets, the basic model used herein contains many of the essential features of these wakes.

The principal finding is that there exists an energy-extraction mechanism, whereby the whisker vibrates following a path known as a slaloming trajectory: approaching, barely touching, and then avoiding oncoming vortices, alternating from side to side as the Kármán street vortices travel near the whisker. This motion is enabled by the ellipselike cross-sectional shape of the whisker, which allows the characteristic low pressure at the Kármán street vortices to provide suction forces that drive the oscillations. The lack 
of a self-induced Kármán street by the whiskers allows the synchronization of the whisker vibrations exclusively with the dominant wake frequency.

A similar slaloming motion was first described for propulsive foils operating within upstream wakes, where it was shown that the slaloming trajectory leads to maximum propulsive efficiency achieved through flow energy extraction and increased thrust (Streitlien et al. 1996). A basic difference between the propulsive foils and our problem is that the purpose of the former is to produce thrust at minimum energy; hence the foil generates strong vortices that combine with the oncoming Kármán street vortices following the rules of vorticity control (Streitlien et al. 1996). For this reason, the foil pitch angle must be controlled in order to achieve proper thrust development (Streitlien et al. 1996). In contrast, the whiskers do not produce thrust and hence the vortices they shed are weak, while their pitch angle need not be controlled; therefore the flow mechanism is achieved passively and over a much wider parametric range.

Another case of slaloming trajectory was found in live trout swimming behind a circular cylinder, where the fish were recorded to actively follow a roughly similar slaloming trajectory to produce thrust by extracting energy from the oncoming von Kármán vortices, also leading to synchronization with the frequency of the vortices (Liao et al. 2003). Remarkably, an anaesthetized trout tethered in the wake of a cylinder within a crossstream was found to synchronize passively the flapping of its body, leading to passive propulsion generating thrust through energy extraction (Beal et al. 2006). Again, there are differences between the trout experiments and the whisker response, such as the formation of significant trailing edge vorticity used for propulsion, and the small aspect ratio of the fish body compared to that of the oncoming vortices for the trout, whereas the vorticity generated by the whiskers is weak, while their aspect ratio is large. However, the slaloming trajectory is a basic flow energy extraction mechanism, significant to all three problems.

As previously noted, there are also differences in this experiment's conditions from those experienced by a seal. A cylinder was chosen as the wake generator because it provided the simplest representation of a wake containing regularly arranged vortices, enabling clear elucidation of the principal sensing mechanism. While actual fish wakes (Müller et al. 1997; Wolfgang et al. 1999; Hanke et al. 2000; Hanke \& Bleckmann 2004) differ in some aspects from cylinder wakes, including more complex vortical structures and a finite span extent, the main features of large-scale periodic vortices are similar. Also, the experiment focused on a single whisker sensor, while seals have dozens of whiskers that can make detection of complex wakes more feasible, but we believe that the underlying flow mechanism remains the same.

Since energy extraction via a slaloming vibration has appeared as a basic mechanism in this and in a number of other problems, it may be important for a variety of technical applications. Most relevant to this study is the full development of whisker-like sensors that enable autonomous underwater vehicles to passively sense their surroundings. The slaloming mechanism could also be designed into energy production devices operating behind bluff cylinders within water and air streams.

\section{Conclusions}

The study of a model problem, the interaction of a model of the harbor seal whisker with the wake of an upstream cylinder with larger diameter, provides the basic fluid mechanisms that live seals may employ when they exhibit their outstanding ability to detect and track the wakes of prey even with a $30 \mathrm{~s}$ late start: In open water the whisker model exhibits very low vibration, almost negligible compared to its width. This is largely 
due to its cross-section, which is elliptical and has its long axis aligned with the incoming flow. The wavy geometry further reduces the excitation forces by de-correlating the vortex shedding. However, once the whisker enters the wake of an upstream cylinder, it encounters a side component of velocity, inducing large amplitude oscillations, of the order of its own width. Also, the whisker frequency coincides with the Strouhal frequency of the upstream cylinder, making estimation of the size and shape of the wake-generating body possible.

This result differs from the case of two interacting cylinders of comparable diameter, wherein the downstream flexibly-mounted cylinder oscillates at a frequency determined by its mass and spring properties, the value of the effective added mass, and the spring constant of the wake, as defined in Assi et al. (2013). That frequency can vary substantially away from both the dominant frequency of the upstream cylinder and the cylinder's natural frequency (Hover \& Triantafyllou 2001; Assi et al. 2013). In this whisker-cylinder flow interaction problem, where a stationary upstream cylinder has significantly larger diameter than the downstream body, taking values equal to $2.5,4$, and 11 times the whisker's diameter, the flexibly mounted downstream body vibrates freely with large amplitude at the Strouhal frequency of the upstream cylinder, following a path that "slaloms" among the vortices of the oncoming wake.

This slaloming motion explains the whisker's sensitivity as it enables significant energy extraction from the flow - a mechanism also seen in the visualizations of live trout swimming behind bluff cylinders (Liao et al. 2003) and the upstream swimming of anaesthetized fish and flapping foils (Beal et al. 2006). The extracted energy drives a large amplitude response locked to the dominant frequency in the wake, even at large distances from the wake-producing cylinder; if the upstream cylinder is removed, the whisker practically ceases to vibrate. This explains the exquisite sensitivity of the whiskers of seals, enabling them to detect the principal features of wakes with velocity fluctuations as low as $1 \mathrm{~mm} / \mathrm{s}$.

This flow detection mechanism illuminates the functionality of these whiskers beyond a role of direct touch sensing. Here we demonstrate detection of a wake up to a distance of 160 whisker diameters downstream of the body. It should be noted that far wakes are known to re-organize their vortical structures creating new dominant frequencies (Williamson 1996); this places limits on the precise detectability of the specific object size.

The use of different sized upstream cylinders as wake generators shows that the whisker's detection ability increases with the size of the wake features. A comparison of the whisker's response with that of different geometries displays the whisker shape's heightened detection ability, particularly at larger distances.

\section{Acknowledgements}

The authors acknowledge with gratitude support by ONR, monitored by Dr. Thomas Swean, Jr. under grant N00014-13-1-0059, the William I. Koch Chair in Marine Technology, the MIT Sea Grant Program, and the Singapore National Research Foundation through the Singapore-MIT Alliance for Research and Technology: Center for Environmental Sensing and Modeling (CENSAM). The authors also thank Kathy Streeter and the New England Aquarium for enabling measurement of whiskers by providing specimens shed by their harbor seals.

\section{REFERENCES}


Ahmed, A. \& Bays-Muchmore, B. 1992 Transverse flow over a wavy cylinder. Physics of Fluids A: Fluid Dynamics (1989-1993) 4 (9), 1959-1967.

Assi, G.R.S. $2014 a$ Wake-induced vibration of tandem and staggered cylinders with two degrees of freedom. Journal of Fluids and Structures 50, 340-357.

Assi, G.R.S. $2014 b$ Wake-induced vibration of tandem cylinders of different diameters. Journal of Fluids and Structures 50, 329-339.

Assi, G.R.S., Bearman, P.W., Carmo, B.S., Meneghini, J.R., Sherwin, S.J. \& Willden, R.H.J. 2013 The role of wake stiffness on the wake-induced vibration of the downstream cylinder of a tandem pair. Journal of Fluid Mechanics 718, 210-245.

Assi, G.R.S., Bearman, P.W. \& Meneghini, J.R. 2010 On the wake-induced vibration of tandem circular cylinders: the vortex interaction excitation mechanism. Journal of Fluid Mechanics 661, 365-401.

Assi, G.R.S., Meneghini, J.R., Aranha, J.A.P., Bearman, P.W. \& Casaprima, E. 2006 Experimental investigation of flow-induced vibration interference between two circular cylinders. Journal of Fluids and Structures 22 (6), 819-827.

BARTh, F.G. 2004 Spider mechanoreceptors. Current opinion in neurobiology 14 (4), 415-422.

Beal, D.N., Hover, F.S., Triantafyllou, M.S., Liao, J.C. \& Lauder, G.V. 2006 Passive propulsion in vortex wakes. Journal of Fluid Mechanics 549, 385-402.

Dahl, J.M., Hover, F.S., Triantafyllou, M.S. \& OAKley, O.H. 2010 Dual resonance in vortex-induced vibrations at subcritical and supercritical reynolds numbers. Journal of Fluid Mechanics 643 (1), 395-424.

Dehnhardt, G., Mauck, B. \& Bleckmann, H. 1998 Seal whiskers detect water movements. Nature 394 (6690), 235-236.

Dehnhardt, G., Mauck, B., Hanke, W. \& Bleckmann, H. 2001 Hydrodynamic trailfollowing in harbor seals (phoca vitulina). Science 293 (5527), 102-104.

Flemming, F. \& Williamson, C.H.K. 2005 Vortex-induced vibrations of a pivoted cylinder. Journal of Fluid Mechanics 522, 215-252.

GaO, Y., Sun, Z., TAN, D.S., Yu, D. \& TAN, S.K. 2014 Wake flow behaviour behind a smaller cylinder oscillating in the wake of an upstream stationary cylinder. Fluid Dynamics Research 46 (2), 025505.

Ginter, C. C., DeWitt, T. J., Fish, F. E. \& Marshall, C. D. 2012 Fused traditional and geometric morphometrics demonstrate pinniped whisker diversity. PLoS ONE 7 (4), e34481.

Grant, R., Wieskotten, S., Wengst, N., Prescott, T. \& Dehnhardt, G. 2013 Vibrissal touch sensing in the harbor seal (phoca vitulina): how do seals judge size? Journal of Comparative Physiology A 199 (6), 521-533.

HANke, W. \& Bleckmann, H. 2004 The hydrodynamic trails of Lepomis gibbosus (centrarchidae), Colomesus psittacus (tetraodontidae) and Thysochromis ansorgii (cichlidae) investigated with scanning particle image velocimetry. Journal of Experimental Biology 207 (9), $1585-1596$.

Hanke, W., Brucker, C. \& Bleckmann, H. 2000 The ageing of the low-frequency water disturbances caused by swimming goldfish and its possible relevance to prey detection. Journal of Experimental Biology 203 (7), 1193-1200.

Hanke, W., Witte, M., Miersch, L., Brede, M., Oeffner, J., Michael, M., Hanke, F., Leder, A. \& Dehnhardt, G. 2010 Harbor seal vibrissa morphology suppresses vortexinduced vibrations. Journal of Experimental Biology 213 (15), 2665-2672.

Hans, H., Miao, J.M. \& Triantafyllou, M.S. 2014 Mechanical characteristics of harbor seal (phoca vitulina) vibrissae under different circumstances and their implications on its sensing methodology. Bioinspiration E Biomimetics 9 (3), 036013.

Hover, F.S. \& Triantafyllou, M.S. 2001 Galloping response of a cylinder with upstream wake interference. Journal of fluids and structures 15 (3), 503-512.

Kıм, J. \& Chог, H. 2005 Distributed forcing of flow over a circular cylinder. Physics of Fluids (1994-present) 17 (3), 033103.

LAM, K. \& LiN, Y. F. 2009 Effects of wavelength and amplitude of a wavy cylinder in cross-flow at low reynolds numbers. Journal of Fluid Mechanics 620, 195-220.

LAM, K.M. \& TO, A.P. 2003 Interference effect of an upstream larger cylinder on the lock-in 
vibration of a flexibly mounted circular cylinder. Journal of fluids and structures 17 (8), 1059-1078.

LAM, K., WANG, F.H, LI, J.Y \& So, R.M.C 2004 Experimental investigation of the mean and fluctuating forces of wavy (varicose) cylinders in a cross-flow. Journal of Fluids and Structures 19 (3), 321 - 334.

Lee, S.J. \& NGuyen, A.T. 2007 Experimental investigation on wake behind a wavy cylinder having sinusoidal cross-sectional area variation. Fluid Dynamics Research 39 (4), 292 304 .

Liao, J.C., Beal, D.N., Lauder, G.V. \& Triantafyllou, M.S. 2003 Fish exploiting vortices decrease muscle activity. Science 302 (5650), 1566-1569.

Miersch, L., Hanke, W., Wieskotten, S., Hanke, F. D., Oeffner, J., Leder, A., Brede, M., Witte, M. \& Dehnhardt, G. 2011 Flow sensing by pinniped whiskers. Philosophical Transactions of the Royal Society B: Biological Sciences 366 (1581), 3077-3084.

Müller, U.K., Van Den Heuvel, B.L.E., Stamhuis, E.J. \& Videler, J.J. 1997 Fish foot prints: morphology and energetics of the wake behind a continuously swimming mullet (chelon labrosus risso). Journal of Experimental Biology 200 (22), 2893-2906.

Murphy, Christin T, Eberhardt, William C, Calhoun, Benton H, Mann, Kenneth A \& MANN, DAVID A 2013 Effect of angle on flow-induced vibrations of pinniped vibrissae. PloS one 8 (7), e69872.

Owen, J. C., Szewczyk, A. A. \& Bearman, P. W. 2000 Suppression of karman vortex shedding. Phys. Fluids 12 (9), S9.

Papaionnnou, G.V., Yue, D.K.P., Triantafyllou, M.S. \& Karniadakis, G.E. 2006 Threedimensionality effects in flow around two tandem cylinders. Journal of Fluid Mechanics 558, 387-413.

SAyers, A.T. \& SABAn, A. 1994 Flow over two cylinders of different diameters: The lock-in effect. Journal of Wind Engineering and Industrial Aerodynamics 51 (1), 43-54.

Schulte-Pelkum, N., Wieskotten, S., Hanke, W., Dehnhardt, G. \& Mauck, B. 2007 Tracking of biogenic hydrodynamic trails in harbour seals (phoca vitulina). Journal of Experimental Biology 210 (5), 781-787.

Solomon, J.H. \& Hartmann, M.J. 2006 Biomechanics: robotic whiskers used to sense features. Nature 443 (7111), 525-525.

Streitlien, K., Triantafyllou, G.S. \& Triantafyllou, M.S. 1996 Efficient foil propulsion through vortex control. AIAA Journal 34 (11), 2315-2319.

Wieskotten, S., Dehnhardt, G., Mauck, B., Miersch, L. \& Hanke, W. $2010 a$ Hydrodynamic determination of the moving direction of an artificial fin by a harbour seal (phoca vitulina). Journal of Experimental Biology 213 (13), 2194-2200.

Wieskotten, S., Dehnhardt, G., Mauck, B., Miersch, L. \& Hanke, W. $2010 b$ The impact of glide phases on the trackability of hydrodynamic trails in harbour seals (Phoca vitulina). Journal of Experimental Biology 213 (21), 3734-3740.

Williamson, C.H.K. 1996 Vortex dynamics in the cylinder wake. Annual review of fluid mechanics 28 (1), 477-539.

Williamson, C.H.K. \& Govardhan, R. 2004 Vortex-induced vibrations. Annual Review of Fluid Mechanics 36 (1), 413-455.

Wolfgang, M.J., Anderson, J.M., Grosenbaugh, M.A., Yue, D.K. \& Triantafyllou, M.S. 1999 Near-body flow dynamics in swimming fish. Journal of Experimental Biology 202 (17), 2303-2327.

Zhang, W., Daichin \& Lee, S.J. 2005 Piv measurements of the near-wake behind a sinusoidal cylinder. Experiments in Fluids 38, 824-832, 10.1007/s00348-005-0981-9. 